Published in "Journal of Antimicrobial Chemotherapy 74(6): 1568-1571, 2019"

which should be cited to refer to this work.

\title{
ZHO-1, an intrinsic MBL from the environmental Gram-negative species Zhongshania aliphaticivorans
}

\author{
Nicolas Kieffer ${ }^{1,2}$, Julia Guzmán-Puche ${ }^{1-3}$, Laurent Poirel ${ }^{1,2,4 *}$, Hyo Jung Kang ${ }^{5}$, \\ Che Ok Jeon ${ }^{5}$ and Patrice Nordmann ${ }^{1,2,4,6}$
}

\begin{abstract}
${ }^{1}$ Medical and Molecular Microbiology Unit, Department of Medicine, Faculty of Science and Medicine, University of Fribourg, Fribourg, Switzerland; ${ }^{2}$ INSERM European Unit (IAME, France), University of Fribourg, Fribourg, Switzerland; ${ }^{3}$ University Hospital Reina Sofia, IMIBIC, University of Córdoba, Córdoba, Spain; ${ }^{4}$ Swiss National Reference Center for Emerging Antibiotic Resistance (NARA), University of Fribourg, Fribourg, Switzerland; ${ }^{5}$ Department of Life Science and Research Center for Biomolecules and Biosystems, Chung-Ang University, Seoul, 156-756, Republic of Korea; ${ }^{6}$ Institute for Microbiology, University of Lausanne and University Hospital Centre, Lausanne, Switzerland
\end{abstract}

${ }^{*}$ Corresponding author. Medical and Molecular Microbiology Unit, Department of Medicine, Faculty of Science and Medicine, University of Fribourg, Chemin du Musée 18, CH-1700 Fribourg, Switzerland. Tel: +41-26-300-9583; E-mail: laurent.poirel@unifr.ch

\begin{abstract}
Objectives: Our aim was to characterize the putative MBL of the environmental strain Zhongshania aliphaticivorans isolated from a marine environment.

Methods: The putative MBL was identified in silico using the NCBI database. The $\beta$-lactamase gene was cloned into different Escherichia coli backgrounds. Kinetic parameters were determined using the purified enzyme.

Results: The enzyme named ZHO-1 shared $51 \%$ amino acid identity with the acquired class B carbapenemases IMP-1, KHM-1 and DIM-1. Expression of the bla $\mathrm{ZHO}_{\mathrm{HO}}$ gene in a susceptible E. coli resulted in a carbapenemase phenotype. Kinetic parameters determined from purified ZHO-1 enzyme showed that it had significant hydrolytic activity against most $\beta$-lactams including penicillins, cephalosporins and carbapenems, with the exception of aztreonam and cefepime.
\end{abstract}

Conclusions: This study adds to the knowledge regarding environmental species as a reservoir of possible clinically relevant MBLs.

\section{Introduction}

MBLs are zinc-dependent enzymes capable of hydrolysing all classes of $\beta$-lactams except monobactams. They constitute a highly diverse family of enzymes and can be categorized into three subclasses, namely B1, B2 and B3. The subclass B1 enzymes are the most important clinically since they comprise MBLs such as IMP-1, NDM-1, SPM-1, KHM-1 and VIM-1/-2, ${ }^{1}$ widely identified in Enterobacteriaceae, Acinetobacter spp. and Pseudomonas spp. The spread of MBL genes is associated with mobile genetic elements such as composite transposons and class 1 integrons. It is well known that environmental bacterial species constitute an important reservoir of antimicrobial resistance genes. ${ }^{2}$ According to a recent metagenomic study, the marine environment in particular constitutes a significant reservoir of MBL producers. ${ }^{3}$

In this study, we characterized a novel subtype B1 MBL showing a significant relationship to the IMP-1 enzyme whose gene was identified on the chromosome of an environmental and marine species named Zhongshania aliphaticivorans.

\section{Materials and methods}

\section{In silico sequence analyses}

The putative bla $\mathrm{ZHO}_{\mathrm{HO}}$ gene was identified using the NCBI blast alignment tool. Phylogenetic analysis was performed using the alignment software SeaView (Prabi, La Doua, France).

\section{Bacterial isolates and susceptibility testing}

Strain Z. aliphaticivorans $S M-2^{\top}$ was cultured on marine agar (Roth AG, Arlesheim, Switzerland) at $30^{\circ} \mathrm{C}$ for $36 \mathrm{~h}$. Antimicrobial susceptibility testing was performed according to the standard disc diffusion method using marine agar plates and Mueller-Hinton (MH) agar plates (Bio-Rad, Cressier, Switzerland) for strain $\mathrm{SM}-2^{\top}$ and the Escherichia coli recombinant strains, respectively, following CLSI recommendations. ${ }^{4}$ MICs were determined by broth microdilution using $\mathrm{MH}$ broth.

\section{Cloning and expression of the $\mathrm{bla}_{\mathrm{ZHO}-1}$ gene}

The bla $a_{\mathrm{ZHO}-1}$ gene was cloned into the PTOPO cloning vector using the pCRblunt TOPO cloning kit (Invitrogen, Illkirch, France) with specific primers 
(ZHO-1-Fw: 5'-GCGCAGCCCAATGTCTATTG-3' and ZHO-1-Rv: 5'GCCGAGCGTCAATTA (TGTC-3'). The resulting recombinant plasmid was electroporated into E. coli TOP10 (pZHO-1-TOP) and into OmpC/OmpF porin-deficient E. coli HB4 (pZHO-1-HB4) strains to analyse the additional effect of porin deficiency on the acquired resistance phenotype.

\section{Purification of $\beta$-lactamase $\mathrm{ZHO}-1$}

Four litres of LB broth supplemented with ampicillin (100 mg/L) was inoculated with E. coli TOP10 carrying pZHO-1-TOP for $24 \mathrm{~h}$ at $37^{\circ} \mathrm{C}$ with shaking. The bacterial culture was centrifuged and the pellet was resuspended in Tris-HCl buffer pH $7.5(100 \mu \mathrm{M})$ and sonicated using a Vibra cell ${ }^{\mathrm{TM}} 75186$ sonicator (Thermo Fisher Scientific). After filtration using a $0.22 \mu \mathrm{m}$ nitrocellulose filter, the crude extract was loaded in a Q-Sepharose column connected to an ÄKTAprime chromatography system (GE Healthcare, Glattbrugg, Switzerland) and eluted with a linear $\mathrm{NaCl}$ gradient. The presence of the $\beta$-lactamase was monitored using nitrocefin $(200 \mu \mathrm{M})$. All positive fractions were pooled and dialysed overnight at $4^{\circ} \mathrm{C}$ against HEPES buffer $(0.1 \mathrm{M}, \mathrm{pH} 7.5)$ supplemented with $\mathrm{ZnSO}_{4}(5 \mu \mathrm{M})$. The protein concentrations were measured using Bradford reagent (Sigma-Aldrich, Buchs, Switzerland). The purity of the enzyme was estimated by SDS-PAGE analysis (GenScript, NJ, USA).

\section{Kinetic measurements}

Kinetic measurements were performed at room temperature in HEPES buffer $\left(0.1 \mathrm{M}, \mathrm{pH}\right.$ 7.5) supplemented with $\mathrm{ZnSO}_{4}(5 \mu \mathrm{M})$ using a UV/visible ULTROSPEC 2100 pro spectrophotometer (Amersham Biosciences, Buckinghamshire, UK).

\section{Determination of the zinc concentration dependence of} ZHO-1

In order to determine the effect of different concentrations of $\mathrm{ZnSO}_{4}$ on the enzymatic activity of ZHO-1, three different conditions were tested: (i) a zinc-deprived condition in the presence of 50 mM EDTA; (ii) a standard condition in the presence of $5 \mu \mathrm{M} \mathrm{ZnSO}_{4}$; and (iii) a zinc-excess condition in the presence of $\mathrm{ZnSO}_{4}$ at $50 \mu \mathrm{M}$. These conditions were tested with three different substrates, namely benzylpenicillin, cefalotin and meropenem.

\section{Results and discussion}

Analysis of the Z. aliphaticivorans SM- $2^{\top}$ genome sequence (accession number: NZ_CP014544) revealed a putative chromosomally encoded MBL gene that was named bla $a_{\mathrm{ZH}-1}$. The GC content of $42.6 \%$ of the gene corresponds to that of chromosomally encoded genes of that species. No mobile element was detected surrounding the gene, suggesting that the bla $a_{\mathrm{ZO}-1}$ gene was constitutive for that species. The bla $a_{\mathrm{ZHO}-1}$ gene encoded a putative $\beta$-lactamase (ZHO-1) sharing $51 \%$ amino acid identity with the following acquired class B1 MBLs: IMP-1 identified originally in Serratia marcescens and Pseudomonas aeruginosa ${ }^{5}, \mathrm{KHM}-1$ in Citrobacter freundii ${ }^{6}$ and DIM-1 in Pseudomonas stutzeri. ${ }^{7}$ Protein alignments of the $\beta$-lactamase $\mathrm{ZHO}-1$ with protein structures of these other class $B$ MBLs revealed the conserved amino acid residues known to be involved in the hydrolytic activity of MBLs such as the histidine residues at positions $116,118,196$ and 263 , the aspartic acid residue at position 120 and the cysteine residue at position 221, respectively [class B $\beta$-lactamase (BBL) nomenclature] (Figure 1). ${ }^{8}$

Antimicrobial susceptibility testing performed with Z. aliphaticivorans strain $\mathrm{SM}-2^{\top}$ demonstrated susceptibility to all $\beta$-lactams. However, once expressed in E. coli TOP10, ZHO-1 conferred high resistance levels to aminopenicillins, carboxypenicillins, and narrowand broad-spectrum cephalosporins including cefalotin, cefoxitin, cefotaxime and ceftazidime, and intermediate susceptibility to ureidopenicillins (such as piperacillin). However, E. coli TOP10 carrying pZHO-1-TOP remained susceptible to cefepime and the monobactam aztreonam. Susceptibility to monobactams is a feature of class B MBL producers. Notably, expression of $\mathrm{ZHO}-1$ in porindeficient E. coli HB4 conferred additional resistance to meropenem and ertapenem, and an intermediate level of resistance to cefepime (Table 1).

The purification of the ZHO-1 enzyme was estimated to be $>90 \%$ with a single dominant band visible on the SDS-polyacrylamide gel. Kinetic parameters of $\mathrm{ZHO}-1$ showed a hydrolytic profile of a class B carbapenemase (Table 2). Actually, strong hydrolytic activities were observed for most $\beta$-lactams tested. The activity

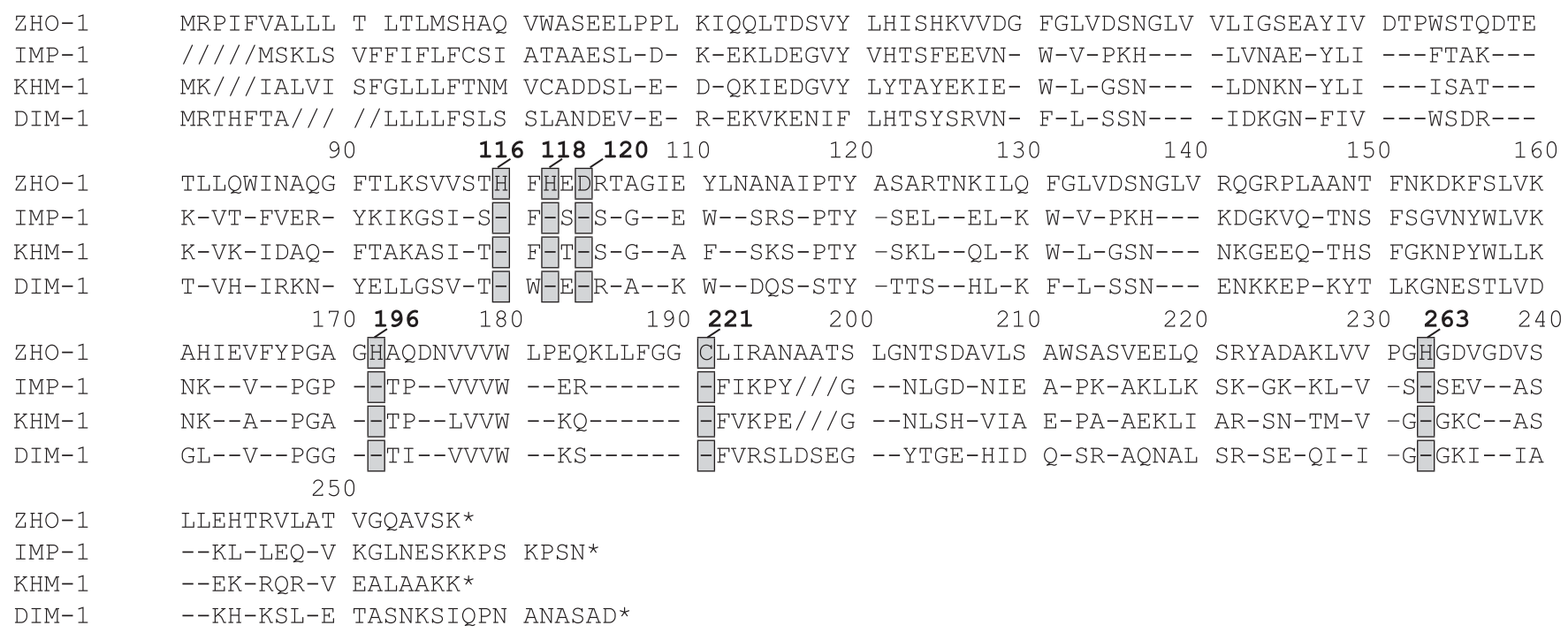

Figure 1. Amino acid sequence comparison between ZHO-1 and its closest MBL relatives. Shaded areas show conserved amino acid residues corresponding to the binding site with the zinc ion required for the hydrolytic activity. Numbers in bold represent the BBL nomenclature. 
Table 1. MICs (mg/L) of $\beta$-lactams for E. coli strains (a WT strain and a porin-deficient strain) with and without the ZHO-1 $\beta$-lactamase gene

\begin{tabular}{|c|c|c|c|c|c|}
\hline Antibiotics & $\begin{array}{l}\text { E. coli TOP10 } \\
\text { (pZHO-1) }\end{array}$ & $\begin{array}{l}\text { E. coli } \mathrm{HB}^{\mathrm{a}}{ }^{\mathrm{a}} \\
(\mathrm{pZZO}-1)\end{array}$ & $\begin{array}{l}\text { E. coli TOP10 } \\
\text { empty vector }\end{array}$ & $\begin{array}{l}\text { E. coli } \mathrm{HB}^{\mathrm{a}}{ }^{\mathrm{a}} \text { empty } \\
\text { vector }\end{array}$ & Z. aliphaticivorans \\
\hline Amoxicillin & 128 & $>128$ & 2 & 16 & 0.5 \\
\hline Amoxicillin/clavulanic acid & 128 & $>128$ & 1 & 16 & 0.5 \\
\hline Piperacillin & $\underline{32}$ & $\underline{64}$ & 1 & 8 & 0.5 \\
\hline Cefalotin & $\overline{128}$ & $>1 \overline{28}$ & 4 & 128 & 0.5 \\
\hline Cefoxitin & 128 & $>128$ & 2 & 64 & 0.5 \\
\hline Ceftazidime & 4 & 128 & 0.12 & 1 & 0.06 \\
\hline Cefotaxime & 64 & 64 & 0.06 & 0.5 & 0.06 \\
\hline Cefepime & 0.5 & $\underline{4}$ & 0.12 & 0.5 & 0.06 \\
\hline Aztreonam & 0.06 & $\overline{0} .5$ & 0.06 & 0.5 & 0.06 \\
\hline Imipenem & 0.5 & 0.5 & 0.06 & 0.06 & 0.06 \\
\hline Meropenem & 1 & 4 & 0.06 & 0.25 & 0.06 \\
\hline Ertapenem & 1 & 4 & 0.06 & 1 & 0.06 \\
\hline
\end{tabular}

Underlined MIC values correspond to intermediate susceptibility and bold MIC values correspond to resistance.

${ }^{a}$ E. coli HB4 is an OmpC/OmpF porin-deficient strain.

( ${ }^{\mathrm{b}}$ Clavulanic acid was added at a fixed concentration of $2 \mathrm{mg} / \mathrm{L}$.

\begin{tabular}{lcrc} 
Table 2. Kinetic parameters of the ZHO-1 MBL \\
\hline Antibiotics & $k_{\text {cat }}\left(\mathrm{s}^{-1}\right)$ & $K_{\mathrm{m}}(\mu \mathrm{M})$ & $k_{\text {cat }} / K_{\mathrm{m}}\left(\mathrm{mM}^{-1} \mathrm{~s}^{-1}\right)$ \\
\hline Penicillin $\mathrm{G}$ & 85 & 150 & 220 \\
Piperacillin & 50 & 1350 & 40 \\
Cefalotin & 4500 & 20 & $>10000$ \\
Cefoxitin & 2 & 150 & 15 \\
Ceftazidime & 1 & 150 & 5 \\
Cefotaxime & 25 & 35 & 0.5 \\
Cefepime & 0.5 & 850 & $\mathrm{ND}$ \\
Aztreonam & $<0.01$ & $>1000$ & 325 \\
Imipenem & 40 & 120 & 500 \\
Meropenem & 10 & 15 & \\
- & & & \\
\hline
\end{tabular}

including carbapenems, but sparing aztreonam and cefepime. Such an enzyme might constitute a potential threat if acquired by clinically relevant species (such as Klebsiella pneumoniae or $P$. aeruginosa). Notably, the $Z$. aliphaticivorans $S M-2^{\top}$ isolate remained susceptible to all $\beta$-lactams despite the presence of the bla $a_{\mathrm{ZH}-1}$ gene on its chromosome. Such a discrepancy might be the consequence of poor expression of this gene in its progenitor. A similar phenomenon has been observed for several environmental species identified as progenitors of carbapenemases, such as Shewanella xiamenensis or Acinetobacter radioresistens, which are the progenitors of the bla $a_{\text {OXA-181 }}$ and bla $a_{\text {OXA-23 }}$ genes, respectively. ${ }^{10,11}$ Finally, these results raise the potential concern of the spread of a hidden reservoir of carbapenemase genes.

\section{Funding}

against broad-spectrum cephalosporins was particularly high, except for cefepime and ceftazidime. Notably, the weak activity observed against ceftazidime was not owing to a lack of affinity considering the very low $\mathrm{K}_{\mathrm{m}}$ value but rather to a poor hydrolysis rate. Noticeably, $\mathrm{ZHO}-1$ also showed significant activity against carbapenems. Finally, no enzymatic activity was detected for aztreonam, which is a common feature of MBLS.

Zinc dependence experiments showed that in the presence of EDTA, the enzymatic activity decreased by $>50 \%$ for each substrate tested. However, no change in activity was observed when increasing the $\mathrm{ZnSO}_{4}$ concentration. These results confirmed that $\mathrm{ZHO}-1$ activity is zinc dependent, a common feature of B1 MBLs.

This study characterized a novel B1 MBL identified from an environmental species recovered from a marine sample. Z. aliphaticivorans $\mathrm{SM}-2^{\top}$ is an environmental species recovered in 2013 from sea tidal flats in the Dangjin coastal area of South Korea. ${ }^{9}$ Members of the Zhongshania genus are Gram-negative, oxidase-positive bacilli, all identified from a marine environment. ZHO-1 showed its ability to hydrolyse most $\beta$-lactams

This work was supported by the Swiss National Science Foundation (project FNS-31003A_163432) and by the University of Fribourg.

\section{Transparency declarations}

None to declare.

\section{References}

1 Cornaglia G, Giamarellou H, Rossolini GM. Metallo- $\beta$-lactamases: a last frontier for $\beta$-lactams? Lancet Infect Dis 2011; 11: 381-93.

2 D'Costa VM, King CE, Kalan L et al. Antibiotic resistance is ancient. Nature 2011; 477: 457-61.

3 Fonseca EL, Andrade BGN, Vicente ACP. The resistome of low-impacted marine environments is composed by distant metallo- $\beta$-lactamases homologs. Front Microbiol 2018; 9: 677.

4 Clinical and Laboratory Standards Institute. Performance Standards for Antimicrobial Susceptibility Testing - Twenty-Eighth Edition: M100. CLSI, Wayne, PA, USA, 2018. 
5 Osano E, Arakawa Y, Wacharotayankun R et al. Molecular characterization of an enterobacterial metallo $\beta$-lactamase found in a clinical isolate of Serratia marcescens that shows imipenem resistance. Antimicrob Agents Chemother 1994; 38: 71-8.

6 Sekiguchi J, Morita K, Kitao T et al. KHM-1, a novel plasmid-mediated metallo- $\beta$-lactamase from a Citrobacter freundii clinical isolate. Antimicrob Agents Chemother 2008; 52: 4194-7.

7 Poirel L, Rodriguez-Martinez JM, Al Naiemi N et al. Characterization of DIM-1, an integron-encoded metallo- $\beta$-lactamase from a Pseudomonas stutzeri clinical isolate in the Netherlands. Antimicrob Agents Chemother 2010; 54: 2420-4.

8 Meini MR, Llarrull LI, Vila AJ. Evolution of metallo- $\beta$-lactamases: trends revealed by natural diversity and in vitro evolution. Antibiotics (Basel) 2014; 3 : 285-316.
9 Lo N, Kang HJ, Jeon CO. Zhongshania aliphaticivorans sp. nov., an aliphatic hydrocarbon-degrading bacterium isolated from marine sediment, and transfer of Spongiibacter borealis Jang et al. 2011 to the genus Zhongshania as Zhongshania borealis comb. nov. Int J Syst Evol Microbiol 2014; 64: 3768-74.

10 Potron A, Poirel L, Nordmann P. Origin of OXA-181, an emerging carbapenem-hydrolyzing oxacillinase, as a chromosomal gene in Shewanella xiamenensis. Antimicrob Agents Chemother 2011; 55: 4405-7.

11 Poirel L, Figueiredo S, Cattoir V et al. Acinetobacter radioresistens as a silent source of carbapenem resistance for Acinetobacter spp. Antimicrob Agents Chemother 2008; 52: 1252-6. 